\title{
Análise de políticas públicas: entre a modelagem e a realidade da política habitacional brasileira'
}

\author{
Paulo Nascimento Neto \\ Pontifícia Universidade Católica do Paraná (PUC-PR) / Programa de Pós-graduação em Gestão \\ Urbana \\ Curitiba / PR - Brasil \\ Tomás Antonio Moreira \\ Instituto de Arquitetura e Urbanismo da USP (São Carlos) / Programa de Pós-Graduação em \\ Urbanismo (PPGU) \\ Campinas / SP - Brasil \\ Carlos Hardt \\ Pontifícia Universidade Católica do Paraná (PUC-PR) / Programa de Pós-graduação em Gestão \\ Urbana \\ Curitiba / PR - Brasil \\ Zulma Schussel \\ Pontifícia Universidade Católica do Paraná (PUC-PR) / Programa de Pós-graduação em Gestão \\ Urbana \\ Curitiba / PR - Brasil
}

\begin{abstract}
Os desafios observados na implementação de políticas públicas têm fomentado uma série de debates no Brasil, entre os quais se destacam aqueles relacionados aos modelos teóricos de policy analysis e seus limites em face da visão multicêntrica e fractal das políticas públicas. Partindo de uma política setorial específica (habitacional), este artigo defende um posicionamento crítico em relação aos estudos monodisciplinares. Contudo, não se pode negar a complexidade dessa empreitada. Compreender os avanços da política habitacional da última década de forma interdisciplinar e integrada constitui uma tarefa intricada e desafiadora. Buscando avançar nesse campo, este artigo tem por objetivos avaliar os modelos teóricos de análise de políticas públicas e, a partir desses elementos, propor constructos com vistas a subsidiar futuros modelos teóricos de análise da política habitacional no Brasil.
\end{abstract}

Palavras-chave: análise de políticas públicas; modelos analíticos; política habitacional.

DOI: http://dx.doi.org/10.1590/0034-7612132489

(cc) BY-NC

Artigo recebido em 26 mar. 2014 e aceito em 27 jan. 2015.

${ }^{1}$ Este trabalho foi desenvolvido com o apoio da Capes através de Bolsa de Doutorado concedida por meio do Programa de Suporte à Pós-Graduação de Instituições de Ensino Particulares (Prosup).

Rev. Adm. Pública - Rio de Janeiro 49(4):847-868, jul./ago. 2015 


\begin{abstract}
Análisis de las políticas públicas: entre el modelado y la realidad de la política de vivienda en Brasil Los desafíos observados en la implementación de políticas públicas han fomentado una serie de debates en Brasil, entre los que podemos destacar los relacionados con los modelos teóricos de análisis de políticas y su limitación frente la perspectiva multicéntrica e fractal de la política pública. De una política específica (política de vivienda de interés social), este artículo defiende una posición crítica en relación a los estudios mono-disciplinares. Sin embargo, no se puede negar la complejidad de esta tarea. La comprensión multidisciplinaria e integrada de los avances de la política de vivienda brasileña desde la última década es una tarea compleja y difícil. Tratando de avanzar en este campo, este artículo tiene como objetivo evaluar los modelos teóricos de análisis de políticas públicas, y de estos elementos, proponer constructos con el fin de apoyar a los futuros modelos teóricos de análisis de la política de vivienda en Brasil.
\end{abstract}

Palabras clave: análisis de políticas públicas; modelos analíticos; política de vivienda.

Analysis of public policies: between modeling and reality of Brazilian housing policy Challenges seen in the implementation of public policies in Brazil have fostered a series of debates, among which we highlight the ones related to the theoretical models of policy analysis and their limitations in face of a polycentric and fractal perspective of public policies. Starting from a specific thematic policy (social housing), this paper sustains a critical point of view about mono-disciplinary studies. However, one cannot deny the complexity of this task. In fact, understand the advances of Brazilian social housing in the last decade, in a multi-disciplinary and integrated way, is a challenge. With the objective to advance in this area, this paper aims to evaluate theoretical models of public policies and, from these elements, propose constructs to support future theoretical models to investigate social housing policy in Brazil.

KEYWORDs: analysis of public policies; theoretical models; housing policy.

\title{
1. Introdução
}

Os desafios observados na implementação de políticas públicas no Brasil têm fomentado uma série de estudos sobre seu processo de elaboração, seus limites e imutabilidades. Para Celina Souza (2003, 2006), o "campo do conhecimento denominado políticas públicas" adquiriu significativo vigor nas últimas décadas, estimulando uma ampla gama de análises sobre as instituições, regras e modelos.

Abordar essa temática suscita debates desde seu aspecto mais fundamental: o próprio conceito de políticas públicas. Conforme afirmam Cavalcanti (2007), Souza (2006) e Bucci (2002), esse termo se caracteriza como uma locução polissêmica cuja conceituação só pode ser aproximada, não existindo "uma única, nem melhor, definição sobre o que [ela] seja" (Souza, 2006:24).

As conceituações, em linhas gerais, enfocam os governos - locus dos conflitos de interesse, ideias e preferências - a partir de uma perspectiva multicêntrica, onde o conjunto possui importância preponderante sobre as partes e os indivíduos, instituições e a interação dos entes governamentais com outros atores constituem elementos fundamentais (Secchi, 
2010; Kooiman, 1993, Rhodes, 1997). Tendo em vista sua característica sintetizante, adotarse-á neste artigo o conceito formulado por Maria Paula Dallari Bucci (2002), que entende políticas públicas como programas de ação governamental visando a coordenação dos meios à disposição do Estado e das atividades privadas para a realização de objetivos socialmente relevantes e politicamente determinados.

Paralelamente à nebulosa conceitual, diversos autores optam por se concentrar em três conceitos-chave: policy, polity e politics. Em linhas gerais, policy (ou public policy) se refere aos conteúdos da política, politics se relaciona aos processos políticos e polity às instituições políticas, ou seja, ao conjunto de procedimentos formais e informais que expressam relações de poder e se destinam à resolução dos conflitos quanto aos bens públicos ${ }^{2}$ (Frey, 2000; Pennings et al., 2006; Zimmermann, 2008; Secchi, 2010).

Essas três categorias analíticas são de grande relevância na ciência política, particularmente na análise de políticas públicas (policy analysis), campo de conhecimento que tem origem nos Estados Unidos na década de 1930, com o objetivo de compreender a forma e as razões pelas quais os governos optam por determinadas ações (Cavalcanti, 2007; Frey, 2000). Para Fischer, Miller e Sidney (2007), os estudos de políticas públicas se caracterizam como uma das áreas das ciências sociais com maior crescimento nas últimas décadas, sendo amplamente utilizados não somente para a compreensão do processo de formulação de políticas públicas, mas também na produção de informações públicas relevantes.

No cenário brasileiro, estudos sobre políticas públicas adquiriram pujança apenas a partir da década de 1990, com a expansão das pesquisas a partir de diversas áreas do conhecimento (Souza, 2003, 2006; Arretche, 2003). Particularmente para o campo habitacional, não se podem negar os avanços significativos obtidos na última década.

A elaboração da Política Nacional de Habitação em 2004, do Sistema Nacional de Habitação em 2005 e do Plano Nacional de Habitação em 2008 estabeleceu um modelo consistente de enfrentamento da questão habitacional no país, fortalecendo a articulação das ações e recursos das diversas esferas de governo e agentes correlatos. Em nível local, os municípios passaram a desenvolver Planos Locais de Habitação de Interesse Social, propondo diretrizes para reverter os problemas associados à irregularidade fundiária e urbanística, precariedade habitacional e carência de novas unidades.

Esse conjunto de instrumentos estabeleceu um novo arcabouço institucional, fortalecendo a articulação das ações, recursos e agentes comprometidos em enfrentar o problema habitacional no Brasil. Entretanto,

(...) ainda que o panorama delineado no país tenha engendrado um processo significativamente positivo de elevação de investimentos no setor habitacional (com grande impulso à produção de

\footnotetext{
${ }^{2}$ Em países de língua latina, como o Brasil, verifica-se uma dificuldade na diferenciação destes termos, visto que os três conceitos-chave são sintetizados na mesma palavra: política (Cavalcanti, 2007; Secchi, 2010).
} 
habitação de interesse social), saltam aos olhos as incongruências entre uma política [nacional de habitação] e um programa habitacional [minha casa minha vida] supostamente convergentes. (...) a Política Nacional de Habitação encontra-se pressionada entre a agenda governamental (sobretudo relacionada à meta quantitativa de novas unidades) e o setor da construção civil, preocupado com a máxima absorção de recursos via construção de um número maior de unidades, sem preocupações urbanísticas e arquitetônicas. (Nascimento Neto, Moreira e Schussel, 2012:92-93)

Os programas habitacionais tendem a transferir o protagonismo de sua efetivação ao mercado imobiliário (construtoras e incorporadas), que possui autonomia excessiva no desenvolvimento do projeto e execução das unidades habitacionais (Shimbo, 2010; Soares et al., 2013). Complementarmente, diversos estudos evidenciam a incapacidade dos municípios em gerir o uso e ocupação do solo, reforçando a segregação socioterritorial e a precariedade ambiental (Rolnik e Klink, 2011; Honda, 2013).

Para Rolnik, Nakano e Cymbalista (2008), a integração entre a política de habitação e a política fundiária é fundamental, permitindo o controle da especulação do solo urbano de forma a evitar a manutenção do padrão fragmentado de urbanização brasileiro, no qual os mais carentes são sistematicamente afastados das áreas ocupadas pelo mercado, demandando investimentos cada vez maiores para sua integração à malha urbana.

Neste contexto, compreender de forma interdisciplinar os avanços da política habitacional brasileira da última década se caracteriza como uma empreitada de difícil consecução. A partir de um extenso estudo bibliométrico ${ }^{3}$ voltado para o campo habitacional, Nascimento Neto e Moreira (2014) ressaltam que a grande maioria das pesquisas apresenta seu escopo centrado em temáticas exaustivamente debatidas, permanecendo em nichos pouco combatíveis, onde a discussão de novos dados à luz de antigas teorias não incorpora elementos inovadores que possam efetivamente contribuir na práxis da gestão pública.

Diante do exposto, impõem-se as seguintes questões-problema: como se avançar na avaliação dos resultados implementados pela Política Nacional de Habitação? Ou, em outras palavras, como avaliar, de forma integral e interdisciplinar, os progressos para a sociedade e para a gestão pública no que tange a políticas habitacionais?

Partindo destas questões, este artigo tem por objetivo avaliar os modelos teóricos de análise de políticas públicas existentes na literatura, identificando seus limites e potencialidades. A partir dessa análise crítica, arrisca-se propor na seção seguinte constructos básicos com vistas a subsidiar futuros trabalhos de construção de modelos teóricos de análise da política habitacional no Brasil. ${ }^{4}$

\footnotetext{
${ }^{3} \mathrm{O}$ estudo bibliométrico compreendeu os trabalhos disponíveis no Banco de Teses e Dissertações da Capes a partir de 1960, os artigos publicados nos periódicos Cadernos Metrópole e Revista Brasileira de Estudos Urbanos e Regionais (RBEUR) e os trabalhos aceitos para comunicação oral no Encontro Nacional da Anpur (Enanpur) na última década. ${ }^{4}$ Com base em extensa pesquisa bibliográfica e em diversos debates realizados dentro dos programas de pós-graduação dos quais os autores fazem parte, este artigo tem um foco eminentemente exploratório, buscando avançar
} 


\section{Análise de políticas públicas}

Os modelos constituem elemento fundamental na análise de políticas públicas, permitindo a representação de concatenações explicativas que pautam a investigação empírica (Frey, 2000), permitindo ao pesquisador entender "why public decisions and their outcomes change, stay stable, vary from sector to sector, and differ in their consequences for the public that consume and appraise them" (John, 2003:482). Dye (2009, 2011) e Sabatier (2007) corroboram o exposto, conceituando o modelo como uma abstração do mundo real para compreender o que é significativo em uma política pública, relacionando constructos e relações.

Tendo em vista a multiplicidade de modelos teóricos existentes, optou-se por analisar três deles, recorrentemente abordados na literatura, a saber: modelo dos fluxos múltiplos, modelo de equilíbrio interrompido e modelo de coalizão de defesa. ${ }^{5}$ Em contraposição aos modelos lineares de tomada de decisão - para os quais determinados fatores geram determinadas decisões - , esses três modelos defendem um processo menos estruturado e determinístico. Conforme ressalta Peters (2002), isso não implica o abandono do planejamento técnico-racional, mas apenas o reconhecimento do papel preponderante da racionalidade limitada dos gestores públicos e de suas habilidades políticas no incremento da performance governamental.

\subsection{Modelo dos fluxos múltiplos}

Formulado por John Kingdon (1995), o modelo de fluxos múltiplos (multiple stream) advoga que as escolhas públicas estão vinculadas às soluções que os tomadores de decisão possuem no momento, considerando que o conhecimento e as informações são limitados e que as instituições públicas operam em um sistema caracterizado por processos decisórios de natureza complexa e simbólica ${ }^{6}$ (Souza, 2006; Cavalcanti, 2007, Secchi, 2010). Dessa forma, as decisões públicas estariam sujeitas à existência de oportunidades e à confluência acidental de problemas, soluções, oportunidades e atores (Capella, 2005; Peters, 2002; John, 2003).

\footnotetext{
em uma concepção alternativa de análise de políticas públicas. Partindo do caso específico da política habitacional, demonstra-se o imperativo de ultrapassar a barreira monodisciplinar e avaliar as políticas públicas de forma integral e interdisciplinar.

${ }^{5}$ Os dois últimos foram reconhecidos como os mais relevantes no campo de políticas públicas na última década, conforme pesquisa realizada pela American Political Science Association (Apsa) (Shoup, 2001).

${ }^{6}$ Segundo Peters (2002), essa concepção não implica o abandono do planejamento racional, mas advoga que a performance governamental está mais relacionada às habilidades políticas dos atores envolvidos do que propriamente a um processo racional de produção de respostas ótimas.
} 
Kingdon (1995) identifica três fluxos: problemas (problems), soluções ou alternativas (policies) e dinâmica política (politics). Na análise do primeiro fluxo (problemas), o autor se concentra nos fatores envolvidos no reconhecimento de um problema e em sua inclusão na agenda governamental. O autor advoga que há um conjunto imenso de problemas e os indivíduos conseguem se concentrar em apenas alguns deles, tendendo a ignorar os demais.

O segundo fluxo (policy stream) engloba o conjunto de soluções disponíveis, não necessariamente motivadas pela percepção de problemas. As ideias seriam geradas em comunidades (policy communities), nas quais aquelas com maior viabilidade técnica e financeira e que exprimem valores amplamente compartilhados perduram. Ao final, o amplo conjunto de ideias é reduzido a um conjunto sintético de alternativas efetivamente consideradas pelo gestor público (Kingdon, 1995).

Kingdon (1995) valoriza de forma recorrente a importância das ideias no processo de definição da agenda. Para o autor, o esforço em tentar compreender as políticas públicas apenas em termos de poder, influência e estratégia conduz à perda de elementos analíticos relevantes. O conteúdo das ideias é visto como parte integrante do processo decisório. Conforme destaca Capella (2005), abordagens como esta ${ }^{7}$ desafiam a perspectiva tradicional de políticas públicas e auxiliam na compreensão da dimensão simbólica desse processo.

O terceiro fluxo (politics stream) envolve a dimensão política, que possui regras próprias e se comporta de forma independente dos problemas socialmente percebidos e das alternativas disponíveis. Nesse fluxo, as coalizões são resultado do processo de negociação política, na qual três elementos se destacam: o clima nacional (national mood), as forças políticas e as mudanças governamentais.

O clima nacional é caracterizado pela convergência da atenção das pessoas em determinadas temáticas, criando um ambiente profícuo para a promoção de algumas questões. As forças políticas, exercidas pelos grupos de pressão, atuam de forma a estimular ou contestar determinadas ideias, podendo tornar o ambiente favorável (ou não) à tomada de decisão. Por fim, as mudanças dentro do próprio governo (alteração em posições estratégicas e mudanças de gestão) tendem a desencadear transformações na agenda política (Kingdon, 1995).

Conforme descrevem Capella (2005) e Weimer e Vining (2011), um conceito fundamental na abordagem de Kingdon é a "janela política”, momento crítico em que ocorre uma convergência entre os três fluxos, gerando uma oportunidade de mudança na agenda. Nesse momento, um problema é identificado, uma solução está disponível e o ambiente político é favorável, possibilitando a tomada de decisão e a criação de uma política pública (figura 1).

\footnotetext{
${ }^{7}$ Para Faria (2003), essas abordagens, classificadas como pós-positivistas, ainda não foram incorporadas de forma consistente na produção acadêmica brasileira.
} 
Figura 1

\section{Representação esquemática do modelo de múltiplas correntes de Kingdon}

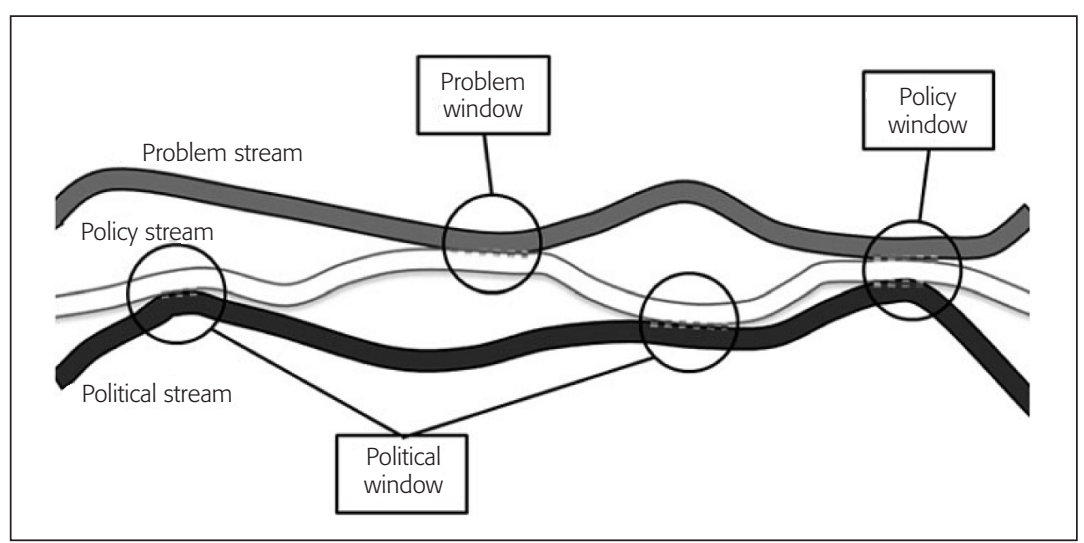

Fonte: Pauli (2011 apud Enserink et al., 2013).

A característica básica da abertura de janelas é seu caráter transitório. As oportunidades de mudança na agenda cessam quando um dos fluxos se desarticula. Conforme afirma Kingdon (1995:165), existem "aberturas de janela" periódicas e previsíveis — como o ciclo orçamentário e o fim do mandato eleitoral - e outras inesperadas. O autor ressalta o papel desempenhado pelos empreendedores de políticas (policy entrepreneurs), dispostos a investir tempo, reputação e dinheiro para defender uma posição em troca de benefícios futuros. Esses indivíduos podem integrar o governo ou situar-se fora dele (grupos de interesse, academia e mídia) e se caracterizam pelo conhecimento de uma questão, pela habilidade em representar as ideias de outros indivíduos ou grupos e manter conexões políticas consistentes.

Os atores influentes no processo de definição da agenda são diferenciados por Kingdon (1995) dentro de dois grupos. O primeiro, composto por atores visíveis, ${ }^{8}$ recebe considerável atenção da imprensa e do público e é influente na definição da agenda governamental, atuando nos fluxos de problemas e política. Já o segundo grupo, formado por atores invisíveis, ${ }^{9}$ forma as comunidades nas quais as ideias são geradas e difundidas, sendo influente na definição das alternativas.

O modelo de fluxos múltiplos tem adquirido relevância no contexto atual da gestão pública, onde a capacidade das autoridades para estruturar decisões de forma independente foi diminuída em favor da ampliação da governança e da gestão participativa, em que as decisões alcançadas são menos previsíveis e vinculadas às bases tradicionais do planejamento racional e tecnocrático (Peters, 2002).

\footnotetext{
${ }^{8}$ Presidente, integrantes do alto escalão governamental, Poder Legislativo, grupos de interesse, partidos políticos, coalizões de campanha, mídia e opinião pública.

${ }^{9}$ Funcionários públicos, analistas de grupos de interesse, assessores parlamentares, acadêmicos, pesquisadores e consultores.
} 
Essa fluidez do modelo em analisar o processo político, ainda que represente um de seus pontos fortes, também é objeto de crítica de diversos autores. Para Mucciaroni (1992), a ênfase no fluxo constante de problemas, soluções e na dinâmica política ignora fatores estruturais que podem interferir de forma determinante na forma como temas são incluídos na agenda. As "regras do jogo" e a estrutura institucional contribuem na determinação dos tipos de problemas identificados pela sociedade, quais deles são considerados responsabilidade do governo e quais os tipos de soluções apropriadas. Contrapondo-se às críticas, Capella (2005) argumenta que a estrutura fluída do modelo e a ausência de relações causa-efeito derivam do próprio conceito do modelo, que se opõe ao determinismo dos sistemas fechados.

\subsection{Modelo do equilíbrio interrompido}

Formulado por Frank Baumgartner e Bryan Jones em 1993, o modelo do equilíbrio interrompido (punctuated equilibrium) defende que as políticas públicas tendem a se manter constantes por longos períodos de estabilidade, interrompidos por curtos períodos de instabilidade onde efetivamente ocorrem transformações significativas nas políticas em curso (Baumgartner e Jones, 2010).

A teoria do equilíbrio interrompido se caracteriza como uma saída ao conflito entre a escola incrementalista e seus críticos, fornecendo um modelo que elucida a existência de períodos de estabilidade pontuados por mudanças expressivas (Robinson, 2006). Segundo afirmam Jones e Baumgartner (2012), seu desenvolvimento foi motivado pela insatisfação dos autores com a capacidade explicativa de os modelos pautados em regras, estabilidade e ajustes incrementais compreenderem as transformações desconexas, episódicas e nem sempre previsíveis nas políticas públicas. Como bem sintetiza John (2006:179-180),

The punctuated equilibrium model connects together in a dynamic framework the various elements of the decision-making. Institutions are important because they freeze a set of political participants into the policy process and exclude others. Institutions help ensure that problems are defined in a particular manner and not in another. (...) Interests and networks are important in the model because, along with the institutional framework, there are patterns of association which help cement agendas and determine the definition of problems. In addition, changes in agendas are often associated with new interest groups and coalitions which may become important in setting the agenda. (...) Individual actors, and their interests, are crucial. The shift in agendas is driven by the way in which the policy system rewards some and excludes others. (...) Ideas are also vital. They are the building-blocks of agendas. Baumgartner and Jones argue that policy-makers and the institutional framework shape the way policy problems are defined.

De forma análoga ao modelo dos fluxos múltiplos, este também se caracteriza por uma abordagem vinculada ao conceito de racionalidade limitada, advogando-se que a es- 
trutura cognitiva dos indivíduos envolvidos é fundamental no processo decisório (Souza, 2006; John, 2003, 2006; Jones e Baumgartner, 2012). Conforme salientam Robinson (2006), Baumgartner e colaboradores (2009), os tomadores de decisões não conseguem avaliar todas as alternativas, pois a busca por soluções demanda tempo e recursos. Nesse contexto, os governos contemporâneos são forçados a gerir uma ampla gama de questões sociais, ambientais e econômicas de natureza concorrente, exigindo uma postura de "malabarista" à medida que organizam a agenda política ante a complexificação das demandas da sociedade.

Essas limitações resultam no levantamento de um conjunto restrito de soluções locais, avaliadas de forma relativa, em relação às alternativas tangíveis e não em relação à totalidade das soluções possíveis. Souza (2006) corrobora o exposto, afirmando que essa capacidade limitada em processar informações conduz os indivíduos a avaliar as questões de forma paralela e não de forma serial (uma de cada vez). Assim, os subsistemas de uma política pública tendem a sofrer mudanças incrementais, baseadas em experiências de implementação e feedback. Somente em períodos de instabilidade, nos quais a atenção se dirige de forma serial a uma temática específica e o ambiente político é propício, mudanças mais profundas ocorreriam.

Neste contexto, o processo de formulação de políticas públicas se insere em um conflito entre as forças de equilíbrio, dominadas por processos de feedbacks negativos, e as forças de desestabilização e contágio, comandadas pelos processos de feedbacks positivos. No primeiro, um distúrbio é acompanhado por ações de compensação, analogamente a um "processo termostático" no qual a temperatura em determinado sistema regula-se automaticamente. Já no segundo, uma mudança bem-sucedida tende a estimular modificações sucessivas, gerando um impulso transformador maior do que o previsto (Jones e Baumgartner, 2012).

Robinson (2006) e Weimer e Vining (2011) alertam para algumas limitações desse modelo. Ao aplicá-la na análise de uma política pública, ainda que a teoria revele especificidades sobre a magnitude de mudanças, os estudos tendem a se concentrar na identificação de padrões e não dos fatores que as motivaram. Para John (2006), as análises não estabelecem relações causais entre as diferentes agendas (opinião pública, mídia, fóruns políticos) e os resultados em termos de políticas públicas. Nesse contexto, o modelo ainda possui limitações na forma como interpreta a influência da opinião pública e da mídia.

Apesar das limitações, intrínsecas a qualquer representação modelar da complexidade social, o modelo do equilíbrio interrompido apresenta grande aplicabilidade como instrumental analítico de compreensão dos movimentos de estabilidade e instabilidade, explorando as tendências de expansão e contração de determinadas temáticas e permitindo efetuar testes consistentes para avaliação de hipóteses. John (2006) corrobora o exposto, afirmando que esse modelo pode ser entendido como parte integrante de uma estrutura analítica maior relacionada à natureza multidimensional da escolha pública, à capacidade limitada dos indivíduos em tomar decisões e à maneira como os sistemas políticos tendem a fixar sua atenção em um pequeno grupo de soluções para os problemas públicos. 


\subsection{Modelo de coalizão de defesa}

O modelo de coalizão de defesa (advocacy coalition framework - ACF) foi desenvolvido por Paul Sabatier e Hank Jenkins-Smith na década de 1980, em resposta às limitações que os autores viam na produção teórica sobre o processo político, centrando suas críticas em três aspectos: (I) a insuficiência explicativa do ciclo político enquanto teoria causal, (II) a necessidade de desenvolver um modelo sistêmico, que integrasse as abordagens top-down e bottom-up e (III) a lacuna dos modelos existentes em considerar a importância do conhecimento técnico-científico no processo político (Souza, 2006; Weible, Sabatier e Mcqueen, 2009). Como resposta, o ACF concedeu um papel de destaque ao conhecimento técnico e científico, criando um modelo sistêmico que integra os estágios do ciclo político e incorpora aspectos de ambas as abordagens de implementação de políticas públicas (top-down e bottom-up) (Sabatier e Jenkins-Smith, 1999).

Para Weible e Sabatier (2007), o ACF se consolidou como um dos modelos mais eficientes, permitindo a compreensão das mudanças nas políticas públicas, particularmente em casos onde há discordância quanto aos objetivos a serem alcançados e às técnicas a serem adotadas, envolvendo múltiplos atores de diferentes níveis governamentais, grupos de interesse, instituições de pesquisa e a mídia. Posteriormente à concepção original da década de 1980, os autores aperfeiçoaram o modelo, que foi objeto de complementações nas décadas de 1990 e 2000 (Sabatier e Jenkins-Smith, 1999; Sabatier, 2007), chegando à forma final apresentada na figura 2 .

A unidade central de análise do modelo é o subsistema da política pública (policy subsystem), que é definido por uma área geograficamente delimitada, uma temática específica e por uma diversidade de participantes de diferentes níveis governamentais, grupos de interesse, instituições acadêmicas e a mídia. Com vistas a influenciar na política pública, os atores se especializam em e agem para formar coalizões de defesa (Weible e Sabatier, 2007; Grin e Loeber, 2007).

Segundo Sabatier e Jenkins-Smith (1999), esses grupos procuram exercer sua influência por meio de cinco estratégias principais, a saber: (I) pressionando legisladores para alterar o orçamento e o poder de determinadas agências por meio de contribuições de campanha e depoimentos; (II) influenciando na definição dos titulares de determinados cargos; (III) tentando alterar a opinião pública por intermédio dos meios de comunicação; (IV) procurando alterar o comportamento do grupo-alvo de determinada política por meio de manifestações ou boicotes; e (V) tentando transformar gradualmente a percepção dos diversos atores envolvidos em uma política pública por meio de pesquisas e troca de informações.

Contudo, o subsistema da política pública não se processa de forma hermética. Ele se desenvolve dentro, é afetado e afeta um contexto mais amplo da sociedade que, dentro do modelo ACF, é abarcado por duas categorias. Essas duas categorias interagem com o subsistema da política pública gerando oportunidades de coalizão de longo prazo e restrições de curto prazo, sendo de grande importância para transformações nas políticas públicas, interferindo na densidade, cooperação entre os integrantes dos grupos de coalizão e na estratégia de negociação para atingir objetivos determinados (Weible, Sabatier e Mcqueen, 2009). 


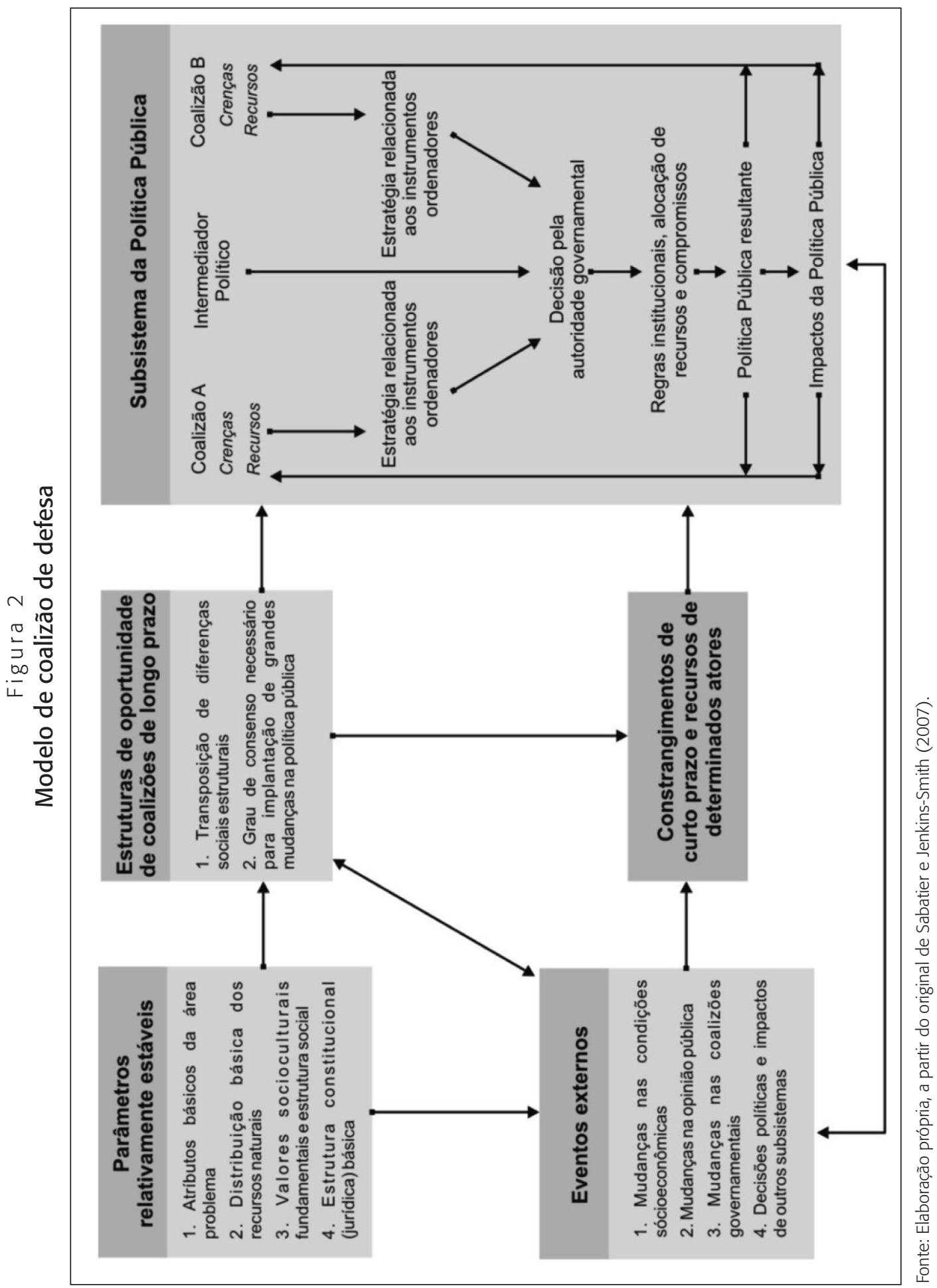


Uma questão central se refere à concepção de indivíduo adotada pelo modelo. De forma semelhante aos modelos anteriormente abordados, o ACF parte do pressuposto de que a racionalidade dos indivíduos é limitada pela restrição de sua capacidade cognitiva em compreender um mundo complexo e adquirir novas informações. Destarte, esses indivíduos tendem a filtrar ou ignorar informações que contestem suas crenças, bem como absorver rapidamente informações que as corroborem. Complementarmente, há uma tendência em recordar as derrotas com maior intensidade - internalizadas como perdas pessoais significativas. Nesse contexto, advoga-se então que os atores são naturalmente motivados a procurar estabelecer uma relação cooperativa com os simpatizantes de suas ideias, formando coalizões de defesa (Weible e Sabatier, 2007).

As crenças dos indivíduos são classificadas em três níveis hierárquicos. O nível mais elevado se refere às crenças profundas (deep core beliefs), que incluem convicções ontológicas e normativas fundamentais que possuem grande resistência à mudança (como a polarização entre direitos individuais $\times$ direitos sociais). O nível intermediário envolve as crenças principais relativas a uma política pública (policy core beliefs), ou seja, crenças empíricas que predominam dentro de um subsistema de política pública (como a adequada distribuição de poder entre governo e mercado). Ainda que menor do que as crenças profundas, também possuem resistência à mudança. Por fim, o nível inferior é composto das crenças secundárias (secondary beliefs), que incluem um conjunto restrito de crenças sobre aspectos específicos do problema da política pública ou de seu contexto. Esse nível é o mais suscetível a mudanças (Sabatier e Jenkins-Smith, 1999; Weible, Sabatier e Mcqueen, 2009).

O ACF utiliza-se destes níveis de crenças para distinguir entre pequenas e substanciais mudanças nas políticas públicas. Conforme expõem Weible e Sabatier (2007), enquanto as mudanças substanciais se relacionam a alterações estruturais no subsistema ou nas crenças principais relativas a uma política pública (policy core beliefs), as pequenas mudanças, de ocorrência mais frequente e menor magnitude, se referem a alterações parciais de âmbito territorial ou material dentro de um subsistema de política pública.

O ACF é considerado um dos modelos teóricos mais debatidos para análise de políticas públicas na atualidade, ${ }^{10}$ sobretudo por sua ampla aplicabilidade em diferentes estruturas de governo, contextos socioculturais e áreas setoriais, e por sua ordenação lógica de subsistemas e fatores que, em última instância, possibilitam uma sistematização clara dos dados, apontando para relações consistentes de causa-efeito (John, 2003; Grin e Loeber, 2007; Weimer e Vining, 2011). Contribuindo com o exposto, Weible e Sabatier (2007) destacam que o modelo evidencia a natureza e a magnitude dos conflitos políticos, advogando que a filiação institucional, ainda que relevante, não é absoluta na construção das coalizões de defesa. Assim, os agentes oficiais, pesquisadores e jornalistas devem ser vistos como membros de diferentes

\footnotetext{
${ }^{10}$ Em âmbito internacional, um levantamento empreendido por Weible, Sabatier e McQueen (2009:136) identificou 80 estudos abrangendo aplicações empíricas do modelo até 2006, revelando "the ACF literature continues to expand (...) and to provide a useful lens for explaining the policy process".
} 
coalizões que, inclusive, podem se encontrar capilarizadas dentro de uma mesma instituição. Essa característica do modelo provê uma flexibilidade maior na análise dos conflitos gerados na formulação de determinada política pública, vinculando os grupos de interesses às crenças e princípios e não unicamente a instituições e organizações.

Para John (2003), a concepção de coalizões de defesa, que se estruturam e se desorganizam ao longo do tempo, é muito mais próxima da realidade contemporânea do que a concepção dos setores políticos como uma rede integrada de atores. Contudo, Weible e Sabatier (2007) alertam que o modelo ACF pode se provar de difícil aplicação, pois o entendimento dos conflitos e mudanças políticas exige uma perspectiva temporal de no mínimo uma década e, tipicamente, demanda a aplicação de questionários e entrevistas. Ambas as demandas consomem recursos financeiros e tempo expressivo do pesquisador.

Por fim, cabe resgatar John (2003), que, corroborado por Jones e Baumgartner (2012), ressalta as similaridades do ACF com o modelo do equilíbrio interrompido, tendo em vista que ambos focam o lado cognitivo e emocional humano do processo de tomada de decisão. Concomitantemente, os pressupostos adotados pelo ACF para compreender as transformações nas políticas públicas também revelam um padrão de processo evolucionário, com períodos de estabilidade pontuados por mudanças estruturais de menor frequência.

\section{Em busca de um modelo integrado de análise de política pública}

As análises empreendidas no item anterior buscam subsidiar as reflexões para construção de um modelo de análise das políticas de habitação que contemple a complexidade das resultantes relacionadas a essa temática. Resgatando as questões norteadoras deste artigo, mais que propor um modelo fechado, procurar-se-á delinear de forma preliminar constructos de análise que possam estruturar futuros modelos analíticos. Neste contexto, Frey (2000) alerta que o instrumental analítico-conceitual disponível na literatura foi desenvolvido em países desenvolvidos, sendo necessária uma adaptação do conjunto de instrumentos às condições peculiares da sociedade brasileira.

De fato, lançar-se à tarefa de propor um modelo de análise — e, ainda, adaptado à realidade nacional — se provou uma tarefa muito complexa e extensa, questão que nos motivou a restringir este artigo à identificação de constructos de mensuração das políticas públicas de habitação. Acreditamos que este constitui um passo inicial basilar, estimulando os debates sobre potenciais modelos de avaliação, sua estrutura fundamental bem como o inter-relacionamento de variáveis.

Nesse contexto, deve-se destacar o esforço de Carlile e Christensen (2005) em sistematizar o processo de formulação de modelos e o desenvolvimento de teorias, estruturando-o dentro de ciclos interativos de teorização (interactive cycles of theory building). Para os autores, o processo (cumulativo) de construção e validação de teorias ocorre em três estágios - observação, categorização e associação - e em ciclos dedutivos e indutivos que se complementam (figura 3). 
Conforme os autores descrevem,

When researchers move from the bottom to the top of the pyramid in these three steps - observation, categorization and association, and in so doing give us constructs, frameworks and models - they have followed the inductive portion of the theory building process. Researchers can then get busy improving these theories by cycling from the top down to the bottom of this pyramid in the deductive portion of the cycle - seeking to test the hypotheses that had been inductively formulated. (...) In contrast to many debates about the virtues of deductive and inductive methods, this suggests that these are two sides to the same pyramid. Every complete lap around the theory building pyramid consists of an inductive side and a deductive side. (Carlile e Christensen, 2005: 4-5)

Figura 3

Processo de construção de teoria

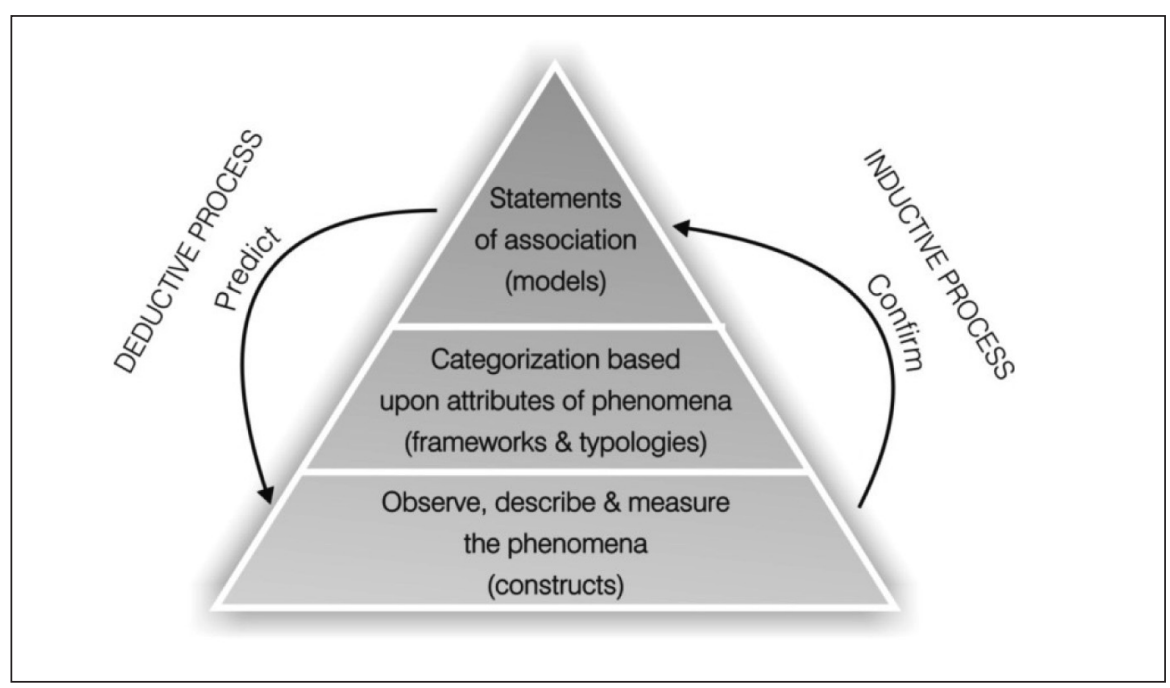

Fonte: Elaboração própria, com base em Carlile e Christensen (2005).

Destarte, o modelo analítico deve resultar do desenvolvimento da primeira volta completa ao redor da pirâmide, onde as reflexões teórico-conceituais, reunidas a partir do referencial teórico e das observações empíricas preliminares do fenômeno, permitirão estabelecer constructos de análise, organizar suas relações em um quadro referencial e construir um modelo teórico de avaliação. Na sequência, a aplicação desse modelo em estudos de caso permite confrontá-lo com a realidade e obter os subsídios necessários para testá-lo e efetuar os ajustes necessários. Essa constitui então a segunda volta ao redor da pirâmide, fornecendo os aportes necessários para a validação e o aumento da consistência do modelo proposto.

Complementarmente, cabe resgatar a classificação proposta por Mayer, Van Daalen e Bots (2004) para os modelos de análise de políticas públicas, tendo em vista sua capacidade de posicionar o escopo do modelo proposto dentro de um contexto mais amplo do campo de 
policy analysis. Para os autores, a análise de políticas públicas é formada por seis grupos principais de atividades, a saber:

1. Pesquisa e análise, relacionada à construção de conhecimento científico e utilização de técnicas de pesquisa (entrevistas, análise estatística etc.);

2. Desenvolvimento e recomendação, voltados à transformação do conhecimento técnico em novas políticas públicas por meio de recomendações e redesenho;

3. Esclarecimento de argumentos e valores, vinculado à identificação dos valores que sustentam um determinado debate político, com vistas a melhorar sua qualidade e encontrar pontos de inconsistência nos grupos de interesse;

4. Provimento de aconselhamento estratégico, buscando propor recomendações vinculadas à análise do contexto e do jogo de poderes envolvidos, com vistas a indicar a um ator específico (geralmente o gestor público) a estratégia mais eficiente para se atingir determinados objetivos;

5. Democratização, função permeada por um posicionamento ético e normativo, que procura construir estratégias para adequar o processo político entre os atores envolvidos, corrigindo iniquidades e garantindo a ampla participação;

6. Mediação, relacionado à análise da relação entre os atores, propondo formas de facilitar encontros onde todas as partes interessadas podem ser consultadas.

A representação diagramática dessas seis atividades é apresentada na figura 4, organizadas segundo seu grau de afinidade. Assim, por exemplo, o desenvolvimento e a recomendação compõem a extensão lógica da pesquisa e análise, bem como o esclarecimento de valores e argumentos fomenta a democratização. Quanto mais distante uma atividade se localizar da outra, maior o nível de tensão para integrá-las (Mayer, Van Daalen e Bots, 2004).

Figura 4

Atividades de análise de políticas públicas e localização do modelo proposto

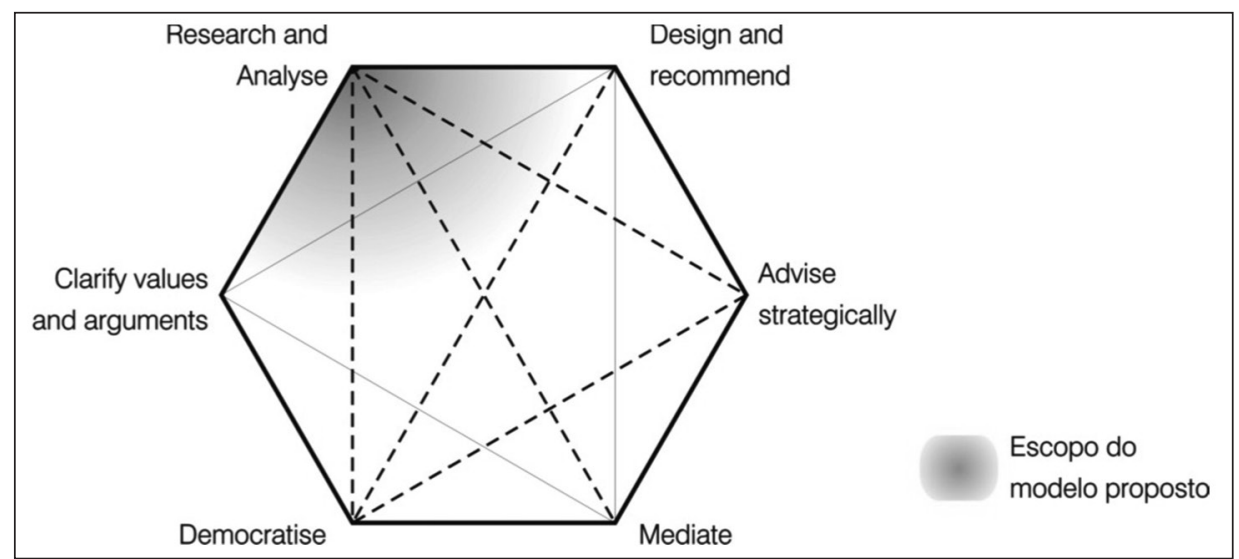

Fonte: Elaboração própria sobre quadro referencial adaptado de Mayer, Van Daalen e Bots (2004). 
Sobreposto ao quadro referencial apresentado, foi incluído o posicionamento desejado do modelo a ser proposto. Como pode ser observado, seu foco principal deve se relacionar com a produção de conhecimento científico com vistas a subsidiar a ampliação dos debates acerca dos limites e avanços das políticas de habitação no Brasil. Complementarmente, como elemento secundário, deve-se estimular a produção de informações que subsidiem o desenho de políticas públicas e permitam identificar os valores utilizados pelos atores sociais envolvidos no processo político.

\section{Política de habitação: perspectivas de análise integrada}

As ideias abordadas buscam instigar o leitor a questionar as formas pelas quais têm se realizado a análise de políticas de habitação no Brasil. Os estudos recorrentemente desenvolvidos, cujos enfoques se concentram no número de unidades construídas e em sua distribuição espacial, são suficientes para avaliar um tema complexo como a habitação social?

Salíngaros e colaboradores (2010:193) corroboram a questão, afirmando que

as pesquisas demonstram amplamente que este modelo não reflete suficientemente o tipo de problema científico que a cidade coloca, pois ele ignora a tremenda complexidade física e social de um tecido urbano de sucesso. De uma maneira inacreditável, ele nem mesmo considera as interações humanas no ambiente construído.

Entendemos que, seja qual for o conjunto de variáveis de análise adotado, um modelo analítico adequado necessita incorporar a preocupação por uma análise interdisciplinar, integrando diferentes aportes teóricos da questão habitacional. Advogamos que é imperativo se partir de um conceito basilar suficientemente abrangente para abarcar a complexidade da temática, requisito que nos conduziu a trabalhar com o desenvolvimento sustentável.

O termo, utilizado pela primeira vez no Relatório Brundtland (ONU, 1987), surge com as intensas discussões sobre o crescimento demográfico orientado em um modelo de desenvolvimento baseado no consumo de bens naturais. Nesse cenário, ele é entendido como "aquele que atende às necessidades do presente sem comprometer a possibilidade das gerações futuras atenderem as suas próprias necessidades" (CMMAD, 1991:46). Esse conceito foi consolidado na Conferência das Nações Unidas sobre Meio Ambiente e Desenvolvimento, realizado no Rio de Janeiro em 1992, onde o Desenvolvimento Sustentável serviu de base para a série de compromissos firmados, entre os quais se destaca a Agenda 21.

Conforme apontando pela ONU (2001), com base na Agenda 21 (CNUMAD, 2001), o desenvolvimento sustentável pode ser organizado segundo quatro dimensões fundamentais, a saber: (I) político-institucional, (II) ambiental, (III) social e (IV) econômica. É sobre essas dimensões que se propõe instigar a academia a construir uma estrutura para análise da política habitacional no Brasil, procurando agregar variáveis para construção de um estudo abrangente e integrado. 
Uma das principais características dessas dimensões é sua difícil separação estanque, tendo em vista a complexidade das relações existentes e o inter-relacionamento de fatores. Essa constatação pode ser confirmada quando tomamos como base a Política Nacional de Habitação, instrumento fundamental da temática no Brasil: a agregação de suas diretrizes segundo as dimensões da sustentabilidade revela que as mesmas envolvem mais de uma delas (tabela 1).

Tabela 1

Diretrizes da Política Nacional de Habitação × dimensões da sustentabilidade

\begin{tabular}{|c|c|c|c|c|}
\hline Política Nacional de Habitação & \multicolumn{4}{|c|}{ Dimensões da Sustentabilidade } \\
\hline Diretrizes & Ambiental & Social & Econômica & Institucional \\
\hline Desenvolvimento institucional & & & & \\
\hline Mobilização de recursos, identificação da demanda e gestão de subsídio & & & & \\
\hline Qualidade e produtividade da produção habitacional & & & & \\
\hline Sistema de Informação, avaliação e monitoramento da habitação & & & & \\
\hline Urbanização de assentamentos precários e produção da habitação & & & & \\
\hline Integração da política habitacional à política urbana & & & & \\
\hline Contrato de financiamento habitacional com desequilíbrio financeiro do SFH & & & & \\
\hline
\end{tabular}

Fonte: Elaboração dos autores.

Partindo do exposto, propomos um fluxograma geral para balizar o desenvolvimento de estudos futuros com vistas a desenvolver o modelo de análise integrada, desejado e defendido pelos autores deste artigo (figura 5). O referido fluxograma procura apresentar de forma gráfica as ideias construídas ao longo do artigo, as questões para as quais são necessários progressos e onde se almeja chegar. Também é possível visualizar, de forma mais clara, o intrincado inter-relacionamento das diferentes dimensões da sustentabilidade abordado na tabela 1.

O elemento-chave de inflexão concentra-se na seta localizada no centro da imagem. Ao se conseguir avançar, do reconhecimento da complexidade do objeto para o delineamento de constructos, estar-se-á efetivamente construindo um modelo consistente de análise integrada da política habitacional. Nesse contexto, este artigo se propôs à missão de provocar os leitores a questionar as limitações das atuais análises empreendidas. Não se pretende desvalorizar seus méritos, mas advogar que avanços epistemológicos e metodológicos são necessários.

Esse entendimento baseia-se nas reflexões de Thomas Kuhn (2011), para quem as revoluções científicas ocorrem de modo descontínuo, em saltos qualitativos, a partir dos quais há quebra dos paradigmas vigentes. Nos intervalos dessas "revoluções científicas", a ciência obedeceria a um relativo dogmatismo, se pautando segundo modelos hegemônicos compostos por conjuntos semelhantes de valores, teorias e métodos. Defendemos que nos encontramos diante desse momento: repensar um novo olhar sobre como nós, como pesquisadores, avaliamos os avanços obtidos pela política habitacional no Brasil. 


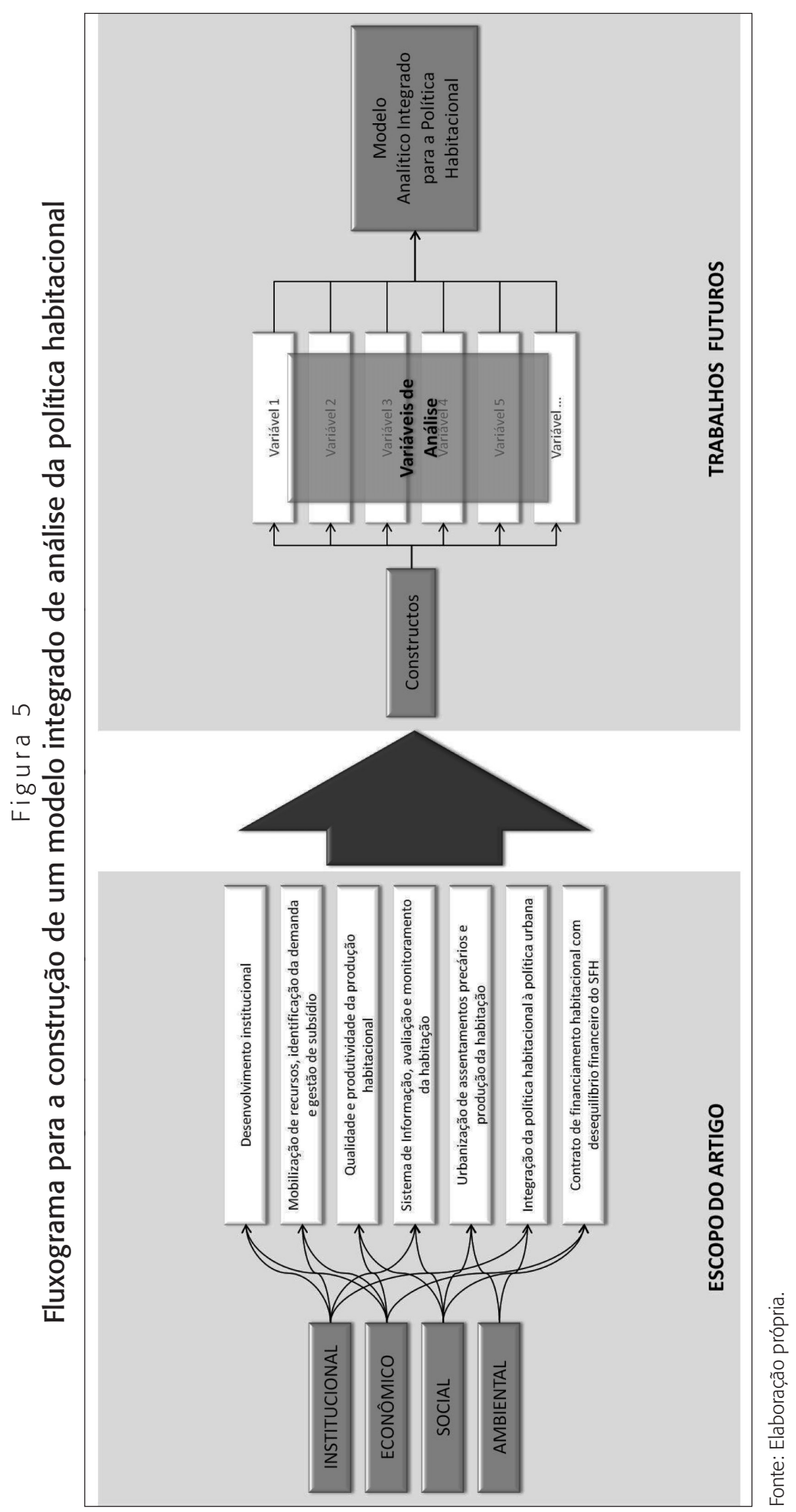


Conforme recorda John (2003), o que distingue os diferentes modelos de avaliação é o pressuposto básico que cada um adota na análise dos processos sociais, que se traduz em um grupo de determinantes e um conjunto esperado de efeitos sociais e econômicos. Assim, ao se transformar a forma como analisamos o objeto política habitacional, será possível identificar impactos e progressos não avaliados até então em sua totalidade, imersos na complexidade das relações sociais, ambientais e econômicas existentes.

\section{Referências}

ARRETCHE, Marta. Dossiê agenda de pesquisa em políticas públicas. Revista Brasileira de Ciências Sociais, v. 18, n. 51, p. 7-10, fev. 2003.

BAUMGARTNER, Frank R.; JONES, Bryan. Agendas and instability in American politics. Chicago: University of Chicago Press, 2010.

BAUMGARTNER, Frank R. et al. Punctuated equilibrium in comparative perspective. American Journal of Political Science, v. 53, n. 3, p. 603-620, jul. 2009.

BUCCI, Maria P. D. Direito administrativo e políticas públicas. São Paulo: Saraiva, 2002.

CAPELLA, Ana Cláudia N. Formação da agenda governamental: perspectivas teóricas. In: ENCONTRO ANUAL DA ANPOCS, XXIX, 2005, Porto Alegre. Anais... Porto Alegre: UFRGS, 2005. p. 1-35.

CARLILE, Paul; CHRISTENSEN, Clayton. Practice and malpractice in management research. Boston: Boston University, 2005.

CAVALCANTI, Paula A. Sistematizando e comparando os enfoques de avaliação e de análise de políticas públicas: uma contribuição para a área educacional. Tese (doutorado em educação) — Universidade Estadual de Campinas, Campinas: 2007.

CNMAD. Comissão Mundial sobre Meio Ambiente e Desenvolvimento. Nosso futuro comum. Rio de Janeiro: Editora FGV, 1991.

CNUMAD. Conferência das Nações Unidas sobre Meio Ambiente e Desenvolvimento, 1992. Agenda 21. Curitiba: Ipardes, 2001.

DOOL, Leon Van den. Making local government work: an introduction to public management for developing countries and emerging economies. Amsterdam: Eburon, 2005.

DYE, Thomas R. Mapeamento dos modelos de análise de políticas públicas. In: HEIDEMANN, Francisco G.; SALM, José Francisco. Políticas públicas e desenvolvimento: bases epistemológicas e modelos de análise. Brasília: UnB, 2009.

DYE, Thomas R. Understanding public policy. Boston: Longman, 2011.

ENSERINK, Bert et al. Policy sciences view on policy analysis. Public Policy Analysis — International Series in Operations Research \& Management Science, v. 179, p. 11-40, 2013. Disponível em: <www. springer.com/social+sciences/book/978-1-4614-4601-9>. Acesso em: 10 jul. 2013. 
FARIA, Carlos A. P. de. A política da avaliação de políticas públicas. Revista Brasileira de Ciências Sociais, v. 20, n. 59, p. 97-110, fev. 2003.

FISCHER, Frank; MILLER, Gerald J.; SIDNEY, Mara S. Handbook of public policy analysis: theory, politics and methods. Nova York: CRC Press, 2007.

FREY, Klaus. Políticas públicas: um debate conceitual e reflexões referentes à prática da análise de políticas públicas no Brasil. Planejamento e Políticas Públicas, n. 21, p. 211-259, jun. 2000.

GRIN, John; LOEBER, Anne. Theories of policy learning: agency, structure, and change. In: FISCHER, Frank; MILLER, Gerald J.; SIDNEY, Mara S. Handbook of public policy analysis: theory, politics and methods. Nova York: CRC Press, 2007. p. 201-222.

HONDA, Sibila C. de A. L. Política habitacional de baixa renda e a atuação do capital privado: o Programa de Arrendamento Residencial em Presidente Prudente (SP). urbe. Revista Brasileira de Gestão Urbana, v. 5, n. 1, p. 107-117, jan./jun. 2013.

JOHN, Peter. Analyzing public policy. Londres: Continuum, 2006.

JOHN, Peter. Is there life after policy streams, advocacy coalitions, and punctuations: using evolutionary theory to explain policy change? The Policy Studies Journal, v. 31, n. 4, p. 481-498, 2003.

JONES, Bryan D.; BAUMGARTNER, Frank R. From there to here: punctuated equilibrium to the general punctuation thesis to a theory of government information processing. The Policy Studies Journal, v. 40, n. 1, p. 1-19, 2012.

KINGDON, John. Agendas, alternatives, and public policies. Nova York: Harper Collins, 1995.

KOOIMAN, Jan. Modern governance: new government — society interactions. Londres: Sage, 1993.

KUHN, Thomas S. A estrutura das revoluções científicas. São Paulo: Perspectiva, 2011.

MAYER, Igor S.; VAN DAALEN, Els; BOTS, Pieter W. G. Perspectives on policy analysis: a framework for understanding and design. International Journal of Technology, Policy and Management, v. 4, n. 2, p. 169-191, 2004.

MUCCIARONI, Gary. The garbage can model and the study of policy making: a critique. Polity, v. 24, n. 3, p. 459-482, 1992. Disponível em: <www.jstor.org/stable/3235165>. Acesso em: 10 jul. 2013.

NASCIMENTO NETO, Paulo; MOREIRA, Tomas. Novas equações para antigas incógnitas: a questão habitacional no Brasil sob a ótica da produção acadêmica nacional. Revista Brasileira de Pós-graduação (Capes), v. 11, n. 24, p. 27, jul. 2014. (no prelo).

NASCIMENTO NETO, Paulo; MOREIRA, Tomas; SCHUSSEL, Zulma. Conceitos divergentes para políticas convergentes: descompassos entre a Política Nacional de Habitação e o Programa Minha Casa Minha Vida. Revista Brasileira de Estudos Urbanos e Regionais (ANPUR), v. 14, p. 83-96, jan./ jun. 2012.

ONU. Organização das Nações Unidas. Indicators of sustainable development: framework and methodologies. Nova York: United Nations Publications, 2001. 
ONU. Organização das Nações Unidas. Our common future. Nova York: United Nations Publications, 1987.

PENNINGS, Paul et al. Doing research in political science. Londres: Sage Publications, 2006.

PETERS, Guy. Governance: a garbage can perspective. Political Science Series, n. 84, p. 1-22, dez. 2002.

RHODES, Roderick A. W. Understanding governance: policy networks, governance, reflexivity and accountability. Philadelphia: Open University Press, 1997.

ROBINSON, Scott E. Punctuated equilibrium models in organizational decision making. In: MORCOL, Goktug (Ed.). Handbook on human decision-making. Flórida: CRC Press, 2006.

ROLNIK, Raquel; KLINK, Jeroen. Crescimento econômico e desenvolvimento urbano: por que nossas cidades continuam tão precárias? Novos Estudos Cebrap, n. 89, p. 89-109, mar. 2011.

ROLNIK, Raquel; NAKANO, K.; CYMBALISTA, R. Participatory master plans: the challenges of democratic management in Brazil: the right of the city. São Paulo: Instituto Pólis; Fundação Ford, 2008.

SABATIER, Paul A. (Ed.). Theories of the policy process. Colorado: Westview Press, 2007.

SABATIER, Paul A.; JENKINS-SMITH, Hank C. The advocacy coalition framework: an assessment. In: SABATIER, Paul A. (Org.). Theories of the policy process. Colorado: Westview Press, 1999. p. 117-168.

SALÍNGAROS, Nikos et al. Habitação socialmente organizada, uma nova abordagem à estrutura urbana I: design capaz de estabelecer posse emocional. urbe. Revista Brasileira de Gestão Urbana, v. 2, n. 2, p. 191-211, jul./dez. 2010.

SECCHI, Leonardo. Políticas públicas: conceitos, esquemas de análise, casos práticos. São Paulo: Cengage Learning, 2010.

SHIMBO, Lucia. Z. Habitação social, habitação de mercado: a confluência entre Estado, empresas construtoras e capital financeiro. Tese (doutorado) - Programa de Doutorado em Arquitetura e Urbanismo, Universidade de São Paulo, São Paulo, 2010.

SHOUP, Brian. Policy section members vote for the most important works in public policy. Policy Currents (Apsa), v. 11, n. 2, p. 14-15, 2011.

SOARES, Isabelle O. et al. Interesses especulativos, atuação do Estado e direito à cidade: o caso do programa "Minha Casa Minha Vida" em Uberaba (MG). urbe. Revista Brasileira de Gestão Urbana, v. 5, n. 1, p. 119-131, jan./dez. 2013.

SOUZA, Celina. Estado do campo da pesquisa em políticas públicas no Brasil. Revista Brasileira de Ciências Sociais, v. 18, n. 16, p. 15-20, fev. 2003.

SOUZA, Celina. Políticas públicas: uma revisão da literatura. Sociologias, Porto Alegre, v. 8, n. 16, p. 20-45, jul./dez. 2006.

WEIBLE, Christopher M.; SABATIER, Paul A. A Guide to the advocacy coalition framework. In: FISCHER, Frank; MILLER, Gerald J.; SIDNEY, Mara S. Handbook of public policy analysis: theory, politics and methods. Nova York: CRC Press, 2007. p. 123-137. 
WEIBLE, Christopher M.; SABATIER, Paul A.; MCQUEEN, Kelly. Themes and variations: taking stock of the advocacy coalition framework. Policy Studies Journal, v. 37, n. 1, p. 121-140, 2009.

WEIMER, David L.; VINING, Aidan. Policy analysis: concepts and practice. Boston: Pearson, 2011.

ZIMMERMANN, Silvia A. Políticas públicas e espaços democráticos. Desenvolvimento em Questão, v. 6, n. 12, p. 11-34, jul./dez. 2008.

Paulo Nascimento Neto é doutor em gestão urbana na Pontifícia Universidade Católica do Paraná (PUC -PR), professor associado do curso de graduação em arquitetura e urbanismo da PCU-PR. E-mail: paulo. nn@uol.com.br.

Tomás Antonio Moreira é doutor em estudos urbanos pela Université du Québec à Montréal, professor associado ao Instituto de Arquitetura e Urbanismo da USP/São Carlos. E-mail: tomas_moreira@hotmail. com.

Carlos Hardt é doutor em meio ambiente e desenvolvimento pela Universidade Federal do Paraná (UFPR), professor associado e coordenador do Programa de Pós-graduação em Gestão Urbana da Pontifícia Universidade Católica do Paraná (PUC-PR).E-mail: c.hardt@pucpr.br.

Zulma Schussel é doutora em meio ambiente e desenvolvimento pela Universidade Federal do Paraná (UFPR), professora associada do Programa de Pós-graduação em Gestão Urbana da Pontifícia Universidade Católica do Paraná (PUC-PR). E-mail: zulma.schussel@pucpr.br. 